\title{
Osmotic shrinkage in star-linear polymer mixtures
}

\author{
A. Wilk ${ }^{1,2}$, S. Huißmann ${ }^{3}$, E. Stiakakis ${ }^{4,5}$, J. Kohlbrecher ${ }^{1}$, D. Vlassopoulos ${ }^{4,6}$, \\ C. N. Likos ${ }^{3,7}$, G. Meier ${ }^{5}$, J. K. G. Dhont ${ }^{5}$, G. Petekidis ${ }^{4,6}$, R.Vavrin ${ }^{1}$
}

1 ETH Zurich \& Paul Scherrer Institut, Laboratory for Neutron Scattering, 5232 Villigen PSI, Switzerland

2 A. Mickiewicz University, Faculty of Physics, Umultowska 85, 61-614 Poznan, Poland

3 Heinrich-Heine-University of Düsseldorf, Department of Physics, 40225

Düsseldorf, Germany

4 FORTH., Institute of Electronic Structure and Laser, 71110 Heraklion, Crete, Greece

5 Forschungszentrum Jülich, Institut für Festkörperforschung, 52425 Jülich, Germany

6 University of Crete, Department of Materials Science \& Technology, 71300

Heraklion, Crete, Greece

7 University of Vienna, Faculty of Physics, Sensengasse 8/12, A-1090 Vienna, Austria.

\begin{abstract}
Multiarm star polymers were used as model grafted colloidal particles with long hairs, to study their size variation due to osmotic forces arising from added linear homopolymers of smaller size. This is the origin of the depletion phenomenon that has been exploited in the past as a means to melt soft colloidal glasses by adding linear chains and analyzed using dynamic light scattering experiments and an effective interactions analysis yielding the depletion potential. Shrinkage is a generic phenomenon for hairy particles, which affects macroscopic properties and state transitions at high concentrations. In this work we present a small angle neutron scattering study of star/linear polymer mixtures with different size ratios (varying the linear polymer molar mass) and confirm the depletion picture, i.e., osmotic star shrinkage. Moreover, we find that as the linear/star polymer size ratio increases for the same effective linear volume fraction (c/c* with $\mathrm{c}^{*}$ the overlapping concentration), the star shrinkage is reduced whereas the onset of shrinkage appears to take place at higher linear polymer volume fractions. A theoretical description of the force balance on a star polymer in solution, accounting for the classic Flory contributions, i.e. elastic and excluded volume, as well as the osmotic force due to the linear chains, accurately predicts the experimental findings of reduced star size as function of linear polymer concentration. This is done in a parameter-free fashion, in which the size of the cavity created by the star, and from which the chains are excluded, is related to the radius of the former from first principles.
\end{abstract}




\section{Introduction}

Star polymers with high functionality have emerged as a novel class of ultrasoft colloidal particles, characterized by interactions that feature Yukawa-type repulsions at long distances and logarithmic repulsions at short distances [1]. They are representative of a large class of long hairy particles, including block copolymer micelles and grafted colloids, where the polymeric nature of the hairs has a stabilizing effect and determines to a large extent their macroscopic behavior [2]. At high volume fraction, achieved at different number densities through manipulation of the effective grafted layer thickness in conditions of varying solvent quality, these systems exhibit a glass-like transition [35]. Its main features are an enhanced frequency-independent storage modulus ( $\left.\mathrm{G}^{\prime}\right)$ in rheology, which is much larger than the loss modulus (G'), and a non-ergodic plateau in the intermediate scattering function [4,5]. Recently, it was observed that small amounts of added linear homopolymer with smaller size compared to the star leads to glass melting due to depletion [4-7]. This osmotic phenomenon [8] is akin to that known in hard colloid-polymer mixtures [9-11] but bears an important difference: the colloidal star is a soft and deformable particle, and thus the osmotic force due to the added polymers can squeeze it, which leads to a shrinkage of its size; for high concentrations eventually star aggregation and possibly phase separation takes place [4,12]. Our previous investigations addressed the depletion problem partly, via dynamic light scattering (DLS) and some limited small angle neutron scattering (SANS) studies, which were complemented by calculations based on an effective-interactions approach $[4,6,7,12]$. However, DLS provided the hydrodynamic radius, whereas the radius of gyration had to be obtained via extrapolation due to the small size of the stars. On the other hand, very limited SANS data are available and there is no systematic study yet on the effect of concentration of added linear chains. Moreover, an important question that has not been addressed is the effect of the linear-to-star polymer size ratio. Last but not least, whereas the depletion effect was unambiguously confirmed with SANS (and rheological) studies, which conformed to the reduced repulsion of the effective pair interaction potential 
$[4,6,13,14,15]$, no theoretical rationalization for the single particle shrinkage from first principles is yet available. Yet, this behavior appears to be generic in soft deformable particles [2,12-16], and a fundamental understanding is needed. Hence, in this work we attempt at complementing our earlier studies by measuring the form factor of single stars in a sea of linear homopolymers of different molar masses, and give a theoretical description of the observed osmotic star shrinkage.

\section{Experimental}

II.1. Materials: We used a model 1,4-polybutadiene star (coded as 12880 , where 128 stands for the nominal star functionality $f$, and 80 for the nominal arm molar mass in $\mathrm{kg} / \mathrm{mol}$ ) with $f=114$ arms and weight-average arm molar mass $72 \mathrm{~kg} / \mathrm{mol}$, synthesized via hydrosilation anionic chemistry [17] and provided by J. Roovers (NRC, Canada). Three deuterated linear 1,4-polybutadienes were used with weight-average molar masses 100,210 and $900 \mathrm{~kg} / \mathrm{mol}$. They were coded as $\mathrm{d}-100, \mathrm{~d}-210$ and d-900, respectively. The d-100 sample was kindly provided by L. Wilner (Forschungszentrum Jülich, Germany) and the other two were obtained from Polymer Source, Canada. We also employed two protonated 1,4-polybutadienes of $165 \mathrm{~kg} / \mathrm{mol}$ (coded as h-165) and $356 \mathrm{~kg} / \mathrm{mol}$ (h-356), obtained by J. Roovers and Polymer Source, respectively. All samples were nearlymonodisperse (the ratio of weight- to number-average molar mass was below 1.1). Solutions in d-cyclohexane, an essentially athermal solvent, were prepared at given concentrations by first preparing a dilute star solution (less than $0.1 \mathrm{wt} \%$ ) and then adding the needed amount of linear polymer under gentle stirring for a few hours. The samples were left to equilibrate for 24 hours before use. A small amount (about $0.1 \mathrm{wt} \%$ ) of antioxidant (4-methyl-2,6-di-tert-butylphenol) was added to reduce the risk of degradation. The radii (hydrodynamic and gyration) of the samples used and the respective hydrodynamic overlapping concentrations $\mathrm{c}_{\mathrm{h}}{ }^{*}$ are listed in Table 1.

II.2. SANS: In all measurements the star concentration was kept low (well below its overlapping value $c^{*}$ ) to ensure conditions for single-particle form factor measurements. 
Samples were placed in $2 \mathrm{~mm}$ thick quartz Hellma cuvettes in the thermostated sample holder (accuracy $\pm 0.1 \%$ ). Data were taken at 3 different detector distances $(1.6,4.5$ and $18 \mathrm{~m})$ and $\lambda=1.20 \pm 0.06 \mathrm{~nm}$ which allows for a variation of the scattering vector $\mathrm{q}$ in the

range $0.01<q\left[(\mathrm{~nm})^{-1}\right]<1$, which is sufficient to follow both the radius of gyration and blob size changes. The raw data (after standard corrections for background, transmission and detector efficiency), were converted into absolute intensities using water as a standard. The background intensity arising from the linear polymers was measured from their solutions without the stars at the experimental conditions set for the samples at the same concentration as for the main sample [18].

\section{Results and discussion}

III.1. SANS data: Fig. 1 depicts typical form factors of the single colloidal star 12880, measured by SANS. In this particular case, the linear homopolymer was d-210 at two concentrations of 0.09 and $5.76 \mathrm{wt} \%$. The data exhibit the typical scattering behavior of multiarm stars [19-22], with two distinct contributions at small and large scattering wave vector $(q)$ values. The low- $q$ part reflects the "form factor" $P$, due to scattering from the overall polymer density profile. The inset of Fig. 1 shows the Guinier representation of the data to show the concentration dependent radii of gyration. The high- $q$ part is dominated by the polymer-solvent fluctuations (blob term) $G$. This leads to the representation of the SANS intensity by two independent contributions [19-22] and was justified by Marques et al. [23] using the Daoud-Cotton model [24].

$I(q)=\mathrm{I}_{0} \frac{1}{f}\left[(f-1) P\left(q R_{g}\right)+G(q \xi)\right]$

where $f$ is the functionality of the star, $R_{g}$ is the radius of gyration and $\xi$ is a correlation length (blob size). Note that the functionality of the star is proportional to the weightaverage star volume. The scattering contribution from the single star is proportional to $f P\left(q R_{g}\right)$ and the contribution from the blob term to $f(f-1) G(q \xi)$ [25]. The above 
representation in eq. 1 leads to the desired normalisation of the forward intensity $I(q=0)=$ $I_{0}$, when $P\left(q R_{g}\right)$ and $G(q \xi)$ are equal to one for $\mathrm{q}=0$. The simplest expression for the form factor is a Guinier type [26]:

$$
P(q)=\exp \left(-q^{2} R_{g}^{2} / 3\right)
$$

The high- $q$ limit $G(q \xi)$ term in equation (1) is approximated by [19]:

$$
G(q \xi)=\frac{\Gamma(\mu)}{q \xi} \frac{\sin \left[\mu \tan ^{-1}(q \xi)\right]}{\left[1+q^{2} \xi^{2}\right]^{\mu / 2}}
$$

where $\mu=\frac{1}{v}-1 \quad(v=3 / 5$ is the Flory exponent), and $\Gamma$ denotes the gamma function. Since the relative contributions of the form factor and the blob contribution are not known, $\Gamma$ will be treated in this analysis as an adjustable parameter, along with $R_{g}$ and $\xi$. The combined fit of Fig. 1 confirms, despite the weak intensity in the high- $q$ regime, the quality of the data and also their good representation with this approach. The same conclusions hold for all measured samples.

The extracted $R_{g}$ values are normalized to the radius of gyration in pure dcyclohexane, $R_{g 0}$ and are compiled in Fig. 2, where they are plotted against the concentration (a) and the effective volume fraction (b) of the added linear homopolymers, the latter being simply the ratio $c / c_{l i n}{ }^{*}$ [27]. A few remarks are in order. First, in this plot we used the hydrodynamic volume fraction $\left(c_{\text {lin }}{ }^{*}\right.$ based on $R_{h}$ ) because it has been measured consistently for all samples, and it allows direct comparison with earlier studies [4]. Given the fact that the ratio of the radius of gyration to the hydrodynamic radius is constant for a polymer-solvent system at fixed temperature, this representation is justified. Furthermore, considering the mixture of 12880 star and h-165 linear homopolymer, it is encouraging that the SANS and light scattering data are virtually identical. This confirms the accuracy of the data and the earlier findings with this mixture 
[4]. Moreover, the reduction of $R_{g} / R_{g 0}$ with $c / c_{\text {lin }}{ }^{*}$ reveals the osmotic effect of the added linear homopolymers in reducing the star's radius of gyration. This effect is unique for soft and deformable objects [7,12-16]. The data of Fig.2a, which are plotted against the weight concentration (or monomer number density), are independent of the linear chain molar mass (Fig. 2a) for the same isotope composition, since the osmotic pressure of the linear polymer solution scales with number density [28]. The latter explains the lower osmotic pressure at the same high $\mathrm{c} / \mathrm{c}_{\text {lin }}{ }^{*}$ for larger linear molar mass.

The plot of Fig. $2 \mathrm{~b}$ suggests that, as the molar mass of the polymer increases, the threshold volume fraction at the onset of star shrinkage (where the ratio $R_{g} / R_{g 0}$ becomes about 0.5 ), increases as well (indicated by vertical arrows in the plot). More specifically, from d-100 to d-210 to d-900, the threshold value appears to shift approximately from 0.3 to 0.8 to 2 , respectively. We note here that whereas the mixtures with linear chains h-165 and $\mathrm{h}-356$ exhibit the same qualitative trend as the other samples, they are very different quantitatively, as demonstrated in Figs. 2(a) and 2(b). In particular, Fig. 2(a) is quite revealing as the $R_{g} / R_{g 0}$ data against concentration are clearly separated in two sets; those with deuterated linear chains and those with protonated linear chains. We attribute this distinct behaviour to the different type of isotope labelling. Indeed, it makes a difference for SANS whether protonated or deuterated linear chains are mixed with the protonated star in deuterated solvent. It is a well-known effect in polymer physics that the exchange of protons with deuterons in a polymer alters its mixing behaviour with other polymers drastically, which is reflected in the vastly different Flory-Huggins parameters [26,29]. Therefore, we will compare quantitatively the shrinking of stars in different linear polymer solutions only for samples with the same isotope composition (i.e., both deuterated solvent and deuterated linear polymer). Of course, since shrinking of the stars is a result of the osmotic pressure due to the presence of the added linear chains, the qualitative effect is observed for all star/linear polymer mixtures, and in this respect the star mixtures with h-165 and h-356 are also discussed in this work. Concerning the latter 
two mixtures, the data confirm the correct qualitative trend, i.e. increasing the molar mass of linear polymer at the same volume fraction reduces the relative shrinking of the star. We note furthermore that the data in Fig. 2(b) suggest that the power-law exponents characterizing the decrease of $R_{g} / R_{g 0}$ with $c / c_{l i n}{ }^{*}$ appear to be similar, and are only slightly larger than the exponent of 0.125 governing the shrinkage of linear and star polymers of low functionality $[1,14]$. Hence, we draw lines with slope 0.125 in this figure. The internal structure of the colloidal stars with arm stretching and reduced monomer density in the periphery $[19,24,30]$ could rationalize a stronger shrinkage, pointing to an effect of functionality. These effects will be quantified below.

\section{III.2. Theoretical analysis:}

The size of a star polymer in a bath of linear chains can be estimated by employing Florytype arguments $[1,28]$ while taking into account the osmotic pressure $\Pi(s)$ exerted on the star due to the presence of the chains. The osmotic pressure depends on the ratio $s \equiv c / c_{\text {lin }}{ }^{*}$ of chain concentration to overlap concentration. The latter is given by $c_{\text {lin }}{ }^{*}=a^{-3} N_{c}{ }^{-3 v}$, where $N_{c}$ denotes the degree of polymerization of the chains, $a$ the length scale of the monomers and $v=3 / 5$ the Flory-parameter.

Insertion of a star polymer in a solution of linear chains, results into the expulsion of the latter from a region of size $R$. The free energy cost for creating such a cavity of radius $R$ in the polymer solution is

$$
\boldsymbol{\beta} F_{o s m}(R)=\frac{4 \pi}{3} R^{3} a^{3} \beta \Pi(s)
$$

where $\beta=1 / k T$ with $\mathrm{k}$ being Boltzmann's coefficient and $R$ is written in reduced units (the length scale is set by the diameter $a$ of the monomer). It should be noted that $R$ need not coincide with the gyration radius of the star, a quantity that characterizes its size, though it is to be expected that the two are very similar. In contrast to the work on hard colloidal particles in polymer solutions [31], we can neglect the surface term here 
because the transition of the polymer density from the excluded region, in the star interior, to its bulk value is gradual [32], minimizing thereby the interfacial cost.

Associated with a star of radius $R_{S}$ with functionality $f$ and degree of polymerization $N_{S}$ is an elastic free energy $\left(F_{e l}\right)$ and excluded volume or interaction free energy $\left(F_{\text {int }}\right)$ which are given by

$\beta F_{e l}\left(R_{s}\right)=\frac{f R_{s}^{2}}{2 N_{s}}$

$\beta F_{\text {int }}\left(R_{s}\right)=v \frac{N_{s}^{2} f^{2}}{2 R_{s}^{3}}$

Again, the star radius $R_{s}$ and the excluded volume parameter $v$ are given in reduced units; we employ, for simplicity, $v=1$ in what follows, which is appropriate for good solvents and maintains the parsimony of the theory in avoiding the usage of several numerical values. The results to follow fall within an order of magnitude, irrespectively of the choice of $v$. We can already see that the competition between the interaction contribution on the one hand and the osmotic and elastic contribution on the other hand, can only lead to a shrinkage of the star with respect to the chain-free case, since the osmotic free energy grows with $R$. Concomitantly, the shrinkage of the stars is a direct consequence of the unbalanced osmotic pressure of the chains, which are depleted from the star's interior. Yet, it remains to be seen whether this mechanism alone is capable of bringing about a satisfactory description of the experimental results, which show quite significant shrinkage of the stars in the presence of homopolymer.

The size of the star is determined as the value at which the sum of the free energies (4)(6) reaches a minimum. Regarding the radius $R$ of the cavity in equation (4) we have to account for the fact that the spatial extent of the region within the star from which the chains are completely excluded (i.e., the cavity), does not necessarily coincide with $R_{s}$, the latter being a measure for the radius of gyration of the star. Employing accurate effective potentials between stars and shorter chains, Camargo and Likos recently showed [32] that the chains around a star penetrate the latter up to a distance $R=\sigma$ from the star center, where $\sigma$ is the so-called corona size of the star, at which the logarithmic star repulsion sets in [1]. Using the well-known relationship $\sigma=4 / 3 R_{s}$, we readily obtain 
$R=b R_{s}$, where $b=1.3$. This numerical factor is practically independent of the size ratio between the chains and the stars, as long as the chains are smaller than the star, and increases slightly with it. It can be anticipated, however, that $b$ attains a larger value when the chains are bigger than the star, since, in that case, the chain would rather surround the star than penetrate it, an issue to which we will return shortly. Hence, we define in what follows $R=b R_{s}$, with the previously mentioned value of $b$.

The size ratio $\delta$ between a chain and a star is defined as $\delta \equiv \frac{R_{c}}{R_{s}}$, where the subscript $c$ denotes the chain and $s$ the star. From the minimization of the free energy with respect to $R_{\mathrm{s}}$ we obtain the following relation, which can be understood as a balance of forces:

$\frac{f R_{s}}{N_{s}}+b^{3} 4 \pi R_{s}^{2} a^{3} \beta \Pi(s)-\frac{3 v N_{s}^{2} f^{2}}{2 R_{s}^{4}}=0$

Within a canonical ensemble renormalization group formalism for a semi-dilute polymer solution a scaling of the osmotic pressure was found having the form [33]

$\frac{\beta \Pi(s)}{c}=1+P(s)$

where

$P(x)=\frac{1}{2} x \exp \left[\frac{1}{4} \varepsilon\left(\frac{\lambda}{x}+\left[\frac{\lambda^{2}}{x^{2}}\right] \ln \lambda+\left[1-\frac{\lambda^{2}}{x^{2}}\right] \ln [x+\lambda]\right)\right]$

and

$x(s)=\frac{1}{2} s \varepsilon \pi^{2}\left[1+\varepsilon\left(\frac{1}{2}+\ln 2\right) / 4\right]$.

The osmotic pressure of a test chain in the solution is given to the order $\varepsilon=4-d$ with the spatial dimensionality $d=3$ in this work and thus $\varepsilon=1$. Further, $\Pi(s)$ depends on the polydispersity, expressed through the index $\lambda$ for which we have $\lambda=1$ since we consider a monodisperse system. The results are practically unchanged for $\lambda<2$. With this information we have $x \cong 6.41 s$.

Noting that

$\mathrm{a}^{3} \beta \Pi(\mathrm{s})=\mathrm{a}^{3} \mathrm{c}[1+\mathrm{P}(\mathrm{s})]=\mathrm{N}_{\mathrm{c}}^{-3 v} \mathrm{~s}[1+\mathrm{P}(\mathrm{s})]=\delta^{-3} \mathrm{~N}_{\mathrm{s}}^{-3 v} \mathrm{f}^{-3 / 5} \mathrm{~s}[1+\mathrm{P}(\mathrm{s})]$, 
where we have used $\delta=\frac{1}{f^{1 / 5}}\left(\frac{N_{c}}{N_{s}}\right)^{v}$, according to scaling theory, the force balance equation (7) can be rewritten as:

$$
\frac{f R_{s}}{N_{s}}+b^{3} 4 \pi R_{s}^{2} \delta^{-3} N_{s}^{-3 v} f^{-3 / 5} s[1+P(s)]-\frac{3 v N_{s}^{2} f^{2}}{2 R_{s}^{4}}=0
$$

The values for the various linear/star ratios $\delta$ are taken from experiment and are listed in Table 2.

Typical predictions of the above equation are depicted in Fig. 3 in the form of normalized radius of gyration against linear polymer $c / c_{l i n}{ }^{*}$, for two cases: fixed linear/star polymer size ratio $\delta=0.34$ and a star of (a) varying functionality and fixed arm size to the experimental value $\left(N_{s}=1393\right)$, or (b) varying arm size and fixed functionality to the experimental value $(f=114)$. In both cases the osmotic effect of added linear chains in shrinking the star is demonstrated. We also note the dramatic effect of functionality on shrinkage, where increasing $f$ at fixed volume fraction reduces the drop of $R_{g}$. This is an effect of the star conformation, in agreement with the experimental findings in Fig.2. On the other hand, the effect of arm size is reduced but again, increasing $N$ reduces the shrinkage at fixed $c / c_{\text {lin }}{ }^{*}$. One can also observe that a drop of the normalized $R_{g}$ of about $10 \%$ is achieved at about $c / c_{l i n}{ }^{*}=1$, pointing to the fact that the osmotic effect of linear chains is clearly important, but of small intensity as a large concentration is needed to achieve a small (10\%) shrinkage. Furthermore, in Fig. 3(b) we see that, in agreement with the experimental findings discussed above, the onset of star shrinking shifts to higher $\mathrm{c} / \mathrm{c}^{*}$ of linear chains as $N_{s}$ increases.

A quantitative comparison of the experimental results with theoretical predictions is shown in Fig. 4 with the $\delta$-values from Table 2. Again, setting aside the samples with protonated linear chains, which show only qualitative agreement with the theory (which can be made quantitative if a different value of $b$ is employed), the results for deuterated linear chains are satisfactory. For all chains considered experimentally, a quantitative agreement is achieved, and moreover the theory is robust given that $b$ is not a fit parameter but rather takes a value that bears microscopic justification on the basis of 
effective chain-chain and chain-star interactions [32,34]. We consider this as an encouraging result that provides predictive power for this important effect for hairy particles. Interesting is the slight disagreement for the case of the d-900 chains, which are indeed bigger than the stars, and for which a larger value of $b$ must be employed, in agreement with the aforementioned anticipation that these penetrate the stars less than shorter ones. In fact, the same argument can be employed for the h-165 and h-356 chains, for which the value of $b=1.3$ underestimates the degree of shrinkage in the same fashion as for the d-900 chains shown in the main plot.

Returning to the experiments, the extracted average star blob size $\xi$ (from Eq. 3 above) is also found to decrease with increasing concentration of the linear polymer. This is depicted in Fig.5. Determination of the blob size is a very delicate issue since the information is hidden in the high- $q$ regime, which is greatly affected by the accuracy of background subtraction; nevertheless, we did find a clear quantitative trend for all solutions measured. This result is consistent with the expectation that the star size variations reflect those of their constituent blobs, and in particular those in the excluded volume regime $[1,24]$. We note that, as in the case with the overall star size $\left(R_{g}\right)$, the shrinkage of $\xi$ for the mixture with h-165 differs from that of mixtures with deuterated linear chains.

\section{Concluding remarks}

The combined experimental and theoretical study of colloidal star-linear homopolymer mixtures in the single star limit, revealed the shrinkage of the former due to the osmotic force exerted by the latter, which is smaller in size. As the linear/star polymer size ratio decreases for the same linear volume fraction, the star shrinkage is enhanced whereas the onset of shrinkage appears to take place at lower linear polymer volume fractions. On the other hand, when plotted against concentration, linear chains of different molar masses give rise to similar star shrinkage due to the same osmotic pressure. Moreover, whereas this osmotic effect is present, its magnitude is rather small, 
as typically the linear chains have to be brought at about their overlap concentration to achieve a reduction in star size of about $10 \%$. At the same time, the outer blobs follow the same trend, as reduction of overall star size is translated into blob size reduction. This phenomenon is generic for soft long hairy particles and provides ways for exploiting depletion as a means for tailoring the state and dynamic behavior of soft colloids. It is also an important molecular parameter that controls macroscopic properties, aside from the stabilizing effect. In closing, we emphasize again the sensitivity of the probed star shrinking behaviour to the different types of isotope labeling of the linear chains. This was observed by comparing the mixture of star with h-165 against the mixtures with deuterated linear chains, and reflected different Flory-Huggins parameters due to different isotope composition of the mixtures.

\section{Acknowledgments}

We are grateful to Jacques Roovers for providing the star and linear h-165 polymers used in this work and for helpful discussions, and to L. Wilner for providing the h-100 polymer. This work was supported in part by the European Union (NoE Softcomp, NMP3-CT2004-502235). The SANS experiments were performed at the Swiss spallation

neutron source SINQ, Paul Scherrer Institute, Villigen, Switzerland. This work was completed when D.V. was visiting the Laboratoire Matière Molle et Chimie at ESPCI in fall 2009 with the support of the "Chaire Michelin-ESPCI".

\section{References}

1. Likos, C. N., Phys. Rep., 348 (2001) 267-439.

2. Vlassopoulos, D., Fytas, G., Adv. Polym. Sci., DOI:10.1007/12 200931 (2009).

3. Vlassopoulos, D., J. Polym. Sci.: Part B: Polym. Phys., 42 (2004) 2931-2941. 
4. Stiakakis, E., Petekidis, G., Vlassopoulos, D., Likos, C. N., Iatrou, H., Hadjichristidis, N., Roovers, J., Europhys. Lett., 72, 664-670 (2005).

5. Helgeson, M. E., Wagner, N. J., Vlassopoulos, D., J. Rheol., 51 (2007) 297-316.

6. Stiakakis, E., Vlassopoulos, D., Likos, C. N., Roovers, J., Meier, G., Phys. Rev. Lett., 89, 208302-1-4 (2002).

7. Stiakakis, E., Vlassopoulos, D., Roovers, J., Langmuir, 19, 6645-6649 (2003).

8. $\quad$ Asakura, S., Oosawa, F., J. Polym. Sci., 33 (1958) 183-192.

9. $\quad$ Sciortino, F., Tartaglia, P., Adv. Phys., 54 (2005) 471-524.

10. Pham, K. N., Puertas, A. M., Bergenholtz, J., Egelhaaf, S. U., Moussaid, A., Pusey, P. N., Schofield, A. B., Cates, M. E., Fuchs, M. and Poon, W. C. K., Science, 296 (2002) 104.

11. Vrij, A., Pure Appl. Chem., 48 (1976) 471-483.

12. Likos, C. N., Mayer, C., Stiakakis, E. and Petekidis, G., J. Phys.: Condens. Matter 17 (2005) S3363-S3369.

13. Dhont, J. K. G., Lettinga, M. P., Dogic, Z., Lenstra, T. A. J., Wang, H., Rathgeber, S., Carletto, P., Willner, L., Frielinghaus, H., Linder, P., Faraday Discuss., 123 (2003) 157.

14. Loppinet, B., Fytas, G., Vlassopoulos, D., Likos, C. N., Meier, G., Liu, G. J., Macromol. Chem. Phys., 206 (2005) 163.

15. A. Fernández-Nieves, Fernández-Barbero, A., Vincent, B., De las Nieves, F. J., J. Chem. Phys. 119, 10383 (2003).

16. B.R. Saunders, Vincent, B., Colloid Polym. Sci. 275, 9 (1997).

17. Roovers, J., Zhou, L.-L., Toporowski, P. M., van der Zwan, M., Iatrou, H., Hadjichristidis, N., Macromolecules, 26 (1993) 4324-4331.

18. Strunz, P., Saroun, J., Keiderling, U., Wiedenmann, A., Przenioslo, R., J. Appl. Cryst. (2000). 33, 829-833

19. Grest, G. S., Huang, J. S., Fetters, L.J., Richter, D., Adv. Chem. Phys., XCIV (1996) 67. 
20. Richter, D., Jucknischke, 0., Willner, L., Fetters, L.J., Lin, M., J.S., Huang, J. S., Roovers, J., Toporovski, C., Zhou, L -L, J. Phys. IV, C8 (1993) 3.;

21. Willner, L., Jucknischke, O., Richter, D., Roovers, J., Zhou, L -L., Toporowski, P. M., Fetters, L. J., Huang, J. S., Lin, M. Y., Hadjichristidis, N., Macromolecules, 27 (1994) 3821.

22. Dozier, W. D. , Huang, J. S., Fetters, L.J., Macromolecules, 24 (1991) 2810.

23. Marques, C. M., Izzo, D., Chariat, T., Mende, E, Eur. Phys. J. B 3 (1998) 353.

24. Daoud, M., Cotton, J. P., J. Phys. (Paris), 43(1982) 531

25. Pedersen, J.S., Gerstenberg, M.C., Macromolecules 29 (1996) 1363-1365.

26. Benoit, H., Higgins, J., Polymers and neutron scattering, Oxford, New York 1994.

27. Roovers, J., Macromolecules, 27 (1994) 5359-5364.

28. Rubinstein, M., Colby, R. H., Polymer Physics, Oxford, New York, 2003.

29. Bates, F. S., Wignall, G. D., Koehler, W. C., Phys. Rev. Lett., 55 (1985) 2425.

30. Semenov, A. N., Vlassopoulos, D., Fytas, G., Vlachos, G., Fleischer, G. , Roovers J., Langmuir, 15 (1999) 358.

31. Louis, A. A., Bolhuis, P. G., Meijer, E. J., Hansen, J. P., J. Chem. Phys., 116 (2002) 10547.

32. Camargo, M., Likos, C. N. submitted for publication (2009).

33. Ohta, T.; Oono, Y. Phys. Lett. 89A, (1982) 460.

34. Mayer, C., Likos, C. N., Macromolecules, 40, (2007) 1196.

\section{Figure captions}

Figure 1: $\quad$ Exemplary form factors of protonated colloidal star 12880 in dilute solution $(0.09 \mathrm{wt} \%)$ in two deuterated solvents, measured by SANS. The solvents consist of linear polybutadiene $\mathrm{d}-210$ in d-cyclohexane: $0.09 \mathrm{wt} \%$ (circles) and $5.76 \mathrm{wt} \%$ (triangles). The solid lines are the Dozier fits (see text). The shrinkage of the star at higher linear polymer concentration is reflected at the low- $-q$ values, whereas slight difference at high- $q$ values reflects the blob size variation. Inset: Guinier plot of the low q-region. 
Figure 2: Plots of the normalized radius of gyration of the star in the linear homopolymer mixture (to its value in pure solvent d-cyclohexane), $R_{g} / R_{g 0}$, as a function of the concentration (a) and the effective volume fraction c/c* (based on $R_{h}$ ) of the polymer (b). SANS data are shown for three linear polybutadienes, d-100 (down triangles), d-210 (filled squares) and d-900 (up triangles). The protonated polybutadiene h-165 (circles) and h-356 (left pointing triangles) data obtained from SANS and light scattering [4] (filled circles) are also shown for comparison. The lines with slope -0.125 in (b) are drawn to suggest the shrinking power-law behaviour (see text). Also in (b), the vertical arrows indicate the onset star shrinking for the solutions with linear d-100, d-210 and d-900 from left to right, respectively.

Figure 3: $\quad$ Radius of gyration $R_{g}$ of a star as a function of linear chain concentration $c / c *$ for a star/linear size ratio of $\delta=0.34$. Shown are the theoretical predictions for (a) fixed degree of polymerization $N_{s}=1393$ and varying functionality from $f=50$ (left curve) to $f=1000$ (right curve) and (b) for fixed functionality $f=114$ and varying arm sizes from $N_{s}=100$ (left curve) to $N_{s}=10000$ (right curve).

Figure 4: Comparison of the theoretical prediction for the degree of shrinkage (selid lines) with the experimental data for the various linear/size ratios $\delta$ from Table 2 . The experimental points are coded as follows: d-34 (circles), d-100 (down triangles), d-210 (filled squares) and d-900 (up triangles). The theoretical results are shown from $\delta=0.19$ (left curve) to $\delta=1.25$ (right curve).

Figure 5: $\quad$ Extracted blob size of the 12880 star in homopolymer solutions (normalized to that of the pure star in the absence of linear chains, $\xi_{0}$ ) as function of the polymer effective (hydrodynamic) volume fraction. Symbols are the same as in Fig.2. 


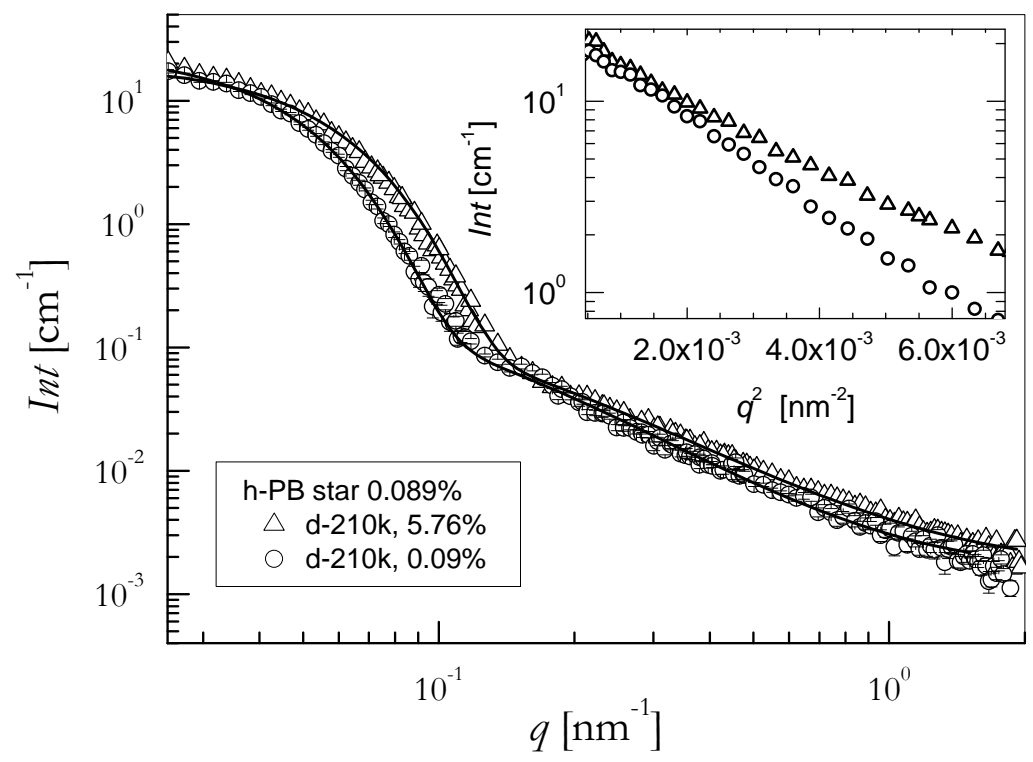

Fig. 1

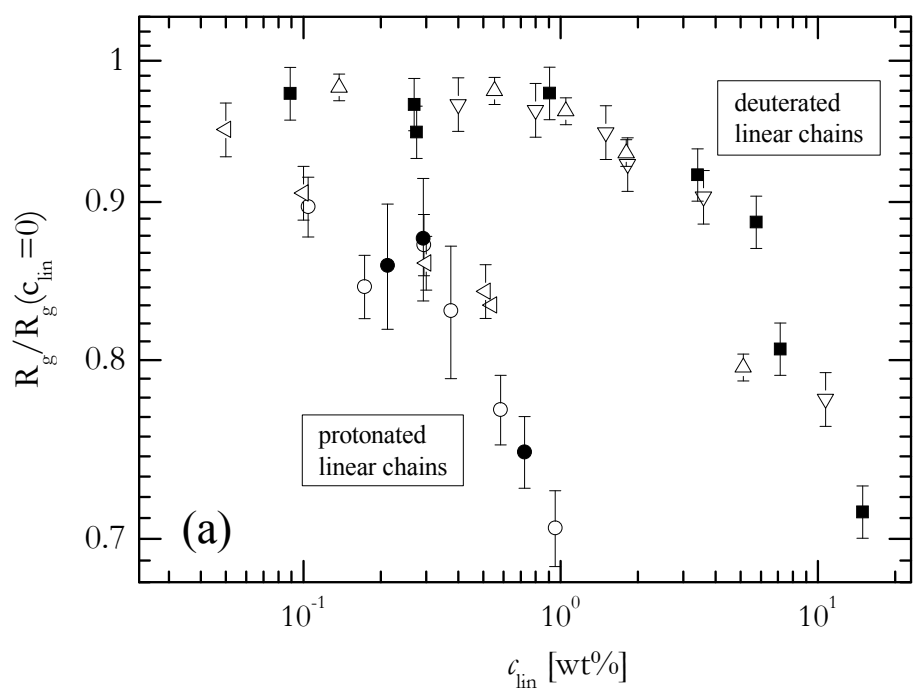

Fig.2(a) 


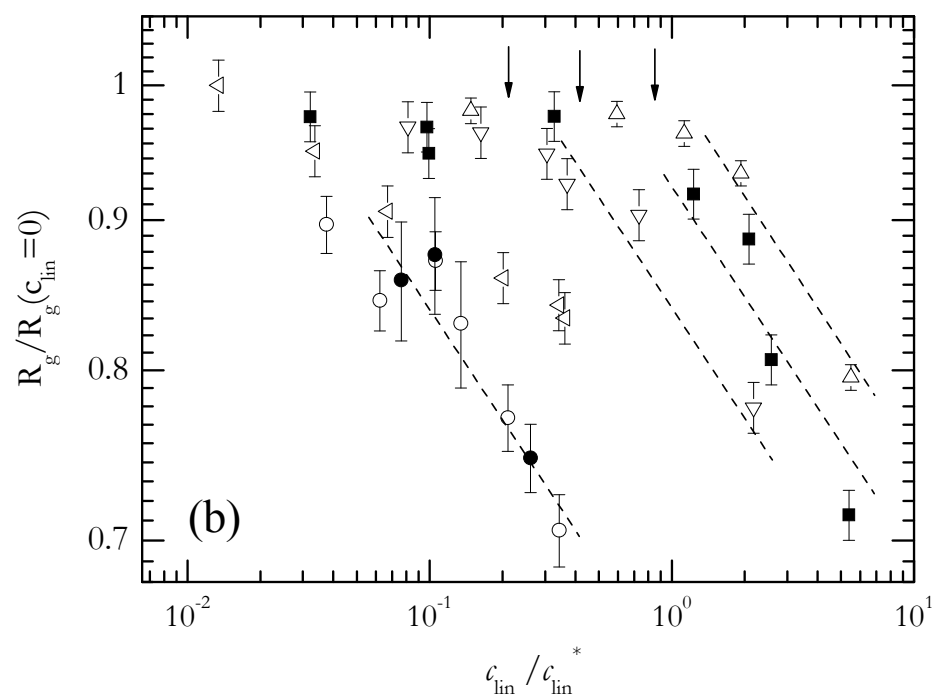

Fig.2(b)

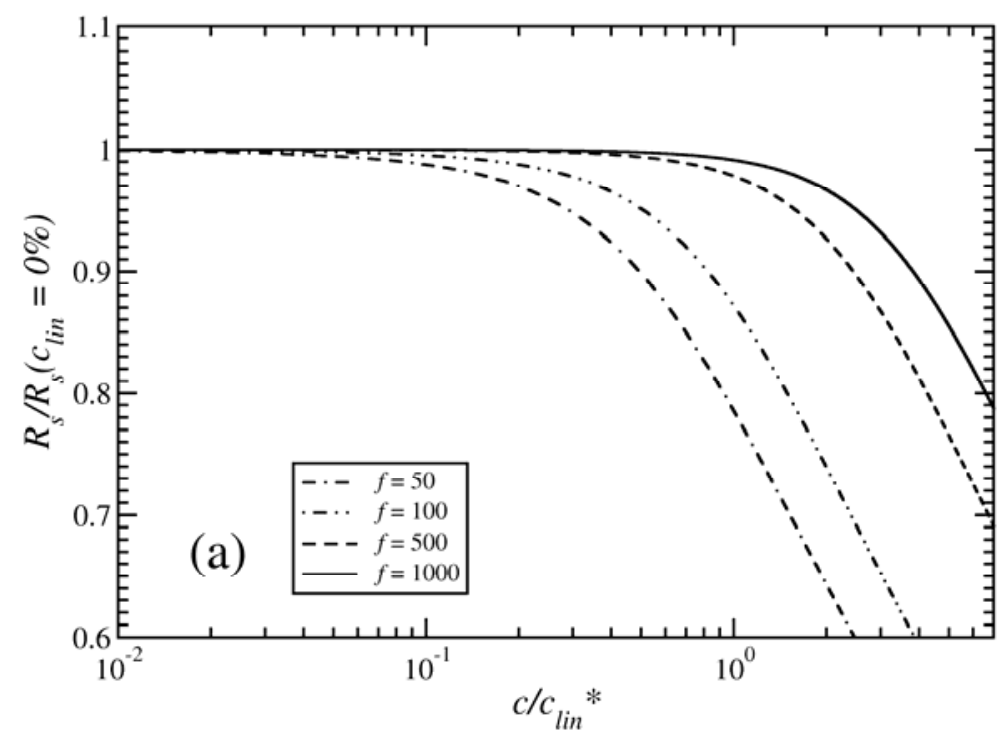

Fig. 3(a) 


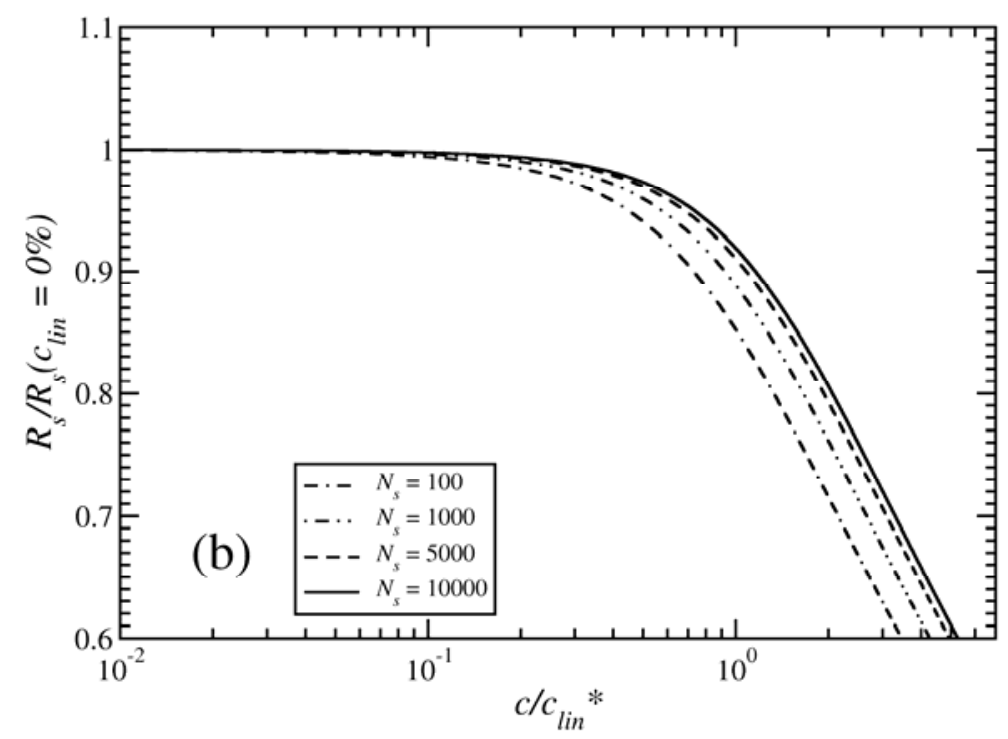

Fig. 3(b)

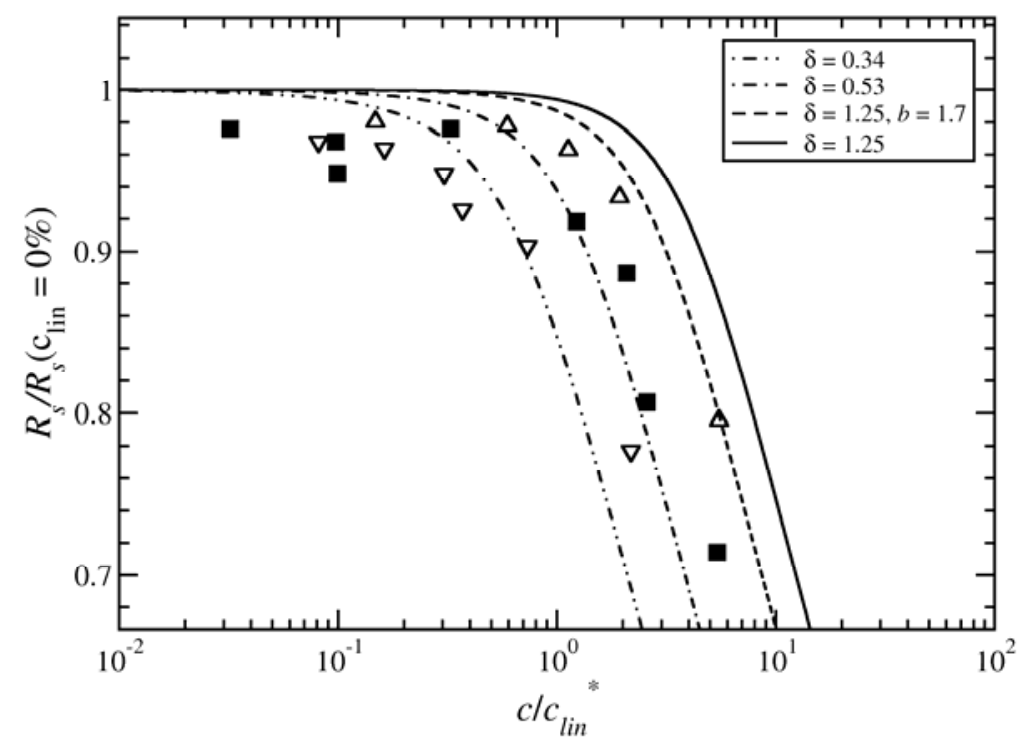

Fig. 4 


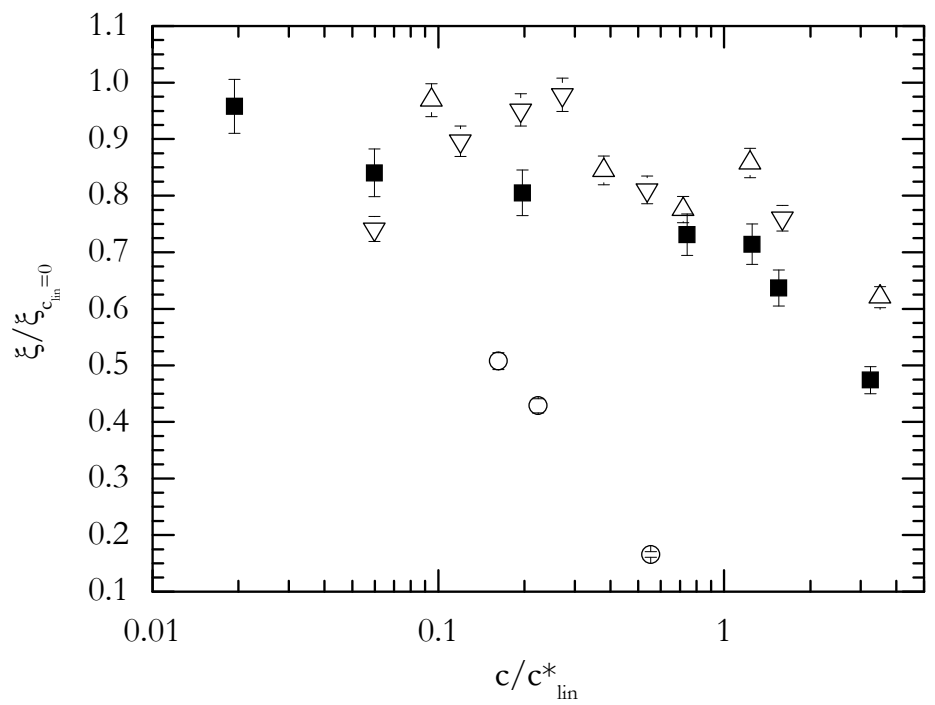

Fig. 5

Table 1: List of samples used in this work along with the hydrodynamic radii, radii of gyration and hydrodynamic overlapping concentration.

\begin{tabular}{|l|l|l|l|}
\hline Sample code & $\mathrm{R}_{\mathrm{h}}[\mathrm{nm}]$ & $\mathrm{R}_{\mathrm{g}}[\mathrm{nm}]$ & $\mathrm{c}_{\mathrm{h}}{ }^{*}[\mathrm{wt} \%]$ \\
\hline \hline $\mathrm{d}-100$ & 9.67 & 13.6 & 4.92 \\
\hline $\mathrm{h}-165$ & 13.8 & 19.5 & 2.78 \\
\hline $\mathrm{d}-210$ & 15.0 & 21.1 & 2.77 \\
\hline h-356 & 21.9 & 30.8 & 1.49 \\
\hline d-900 & 35.0 & 49.9 & 0.93 \\
\hline h-12880 (star) & 66.8 & 41.0 & 1.27 \\
\hline
\end{tabular}


Table 2: Size ratio $\delta$ between the chains and the 12880 star with functionality $f=114$ and degree of polymerization $N_{s}=1393$.

\begin{tabular}{|l|l|}
\hline Sample code & $\delta$ \\
\hline d-100 & 0.34 \\
\hline d-210 & 0.53 \\
\hline d-900 & 1.25 \\
\hline
\end{tabular}

